\title{
starican三חт三
}

Rivista del Dipartimento di Storia Culture Civiltà

Alma Mater Studiorum Università di Bologna

STEFANO TROPEA

Franca Landucci Gattinoni, Filippo re dei Macedoni, Bologna, Il Mulino, 2012, $161 \mathrm{pp}$.

DOI: $10.12977 /$ stor503

ISSN: $1825-411 \mathrm{X}$

Art. $\mathrm{N}^{\circ} 50$

Issue $N^{\circ} 9$ - 2013

Publisher: BraDypUS

December 20 2013

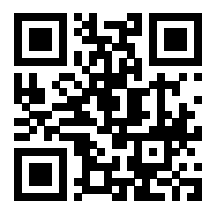





\section{Franca Landucci Gattinoni, Filippo re dei Macedoni, Bologna, II Mulino, 2012, 161 pp.}

\section{STEFANO TROPEA}

Con questo snello libro Franca Landucci Gattinoni, studiosa esperta di storia macedone ed ellenistica, si propone di introdurre brevemente, ma in maniera quanto più possibile accurata e completa, gli eventi che segnarono l'ascesa al trono, l'esercizio del potere e la morte del sovrano Filippo II di Macedonia (359-336 a.C.), distaccandosi da quella tradizione «deformata» (p. 7) che ritiene il regno di Filippo quasi esclusivamente come una naturale premessa alle successive imprese del figlio Alessandro e preoccupandosi di analizzare, con dovizia di particolari e fornendo una breve e pregnante bibliografia di riferimento, la strategia che regolò l'azione politica di questo grande sovrano della casata argeade. In un ristretto numero di parole cariche di significato l'autrice condensa la narrazione di un periodo cruciale nel passaggio dall'età classica all'epoca ellenistica, caratterizzato dalla supremazia macedone sull'intera ecumene ellenica, e sceglie di descrivere questa complessa fase storica componendo una brillante biografia sullo «statista che in poco più di vent'anni spostò il baricentro della Grecia da sud a nord, trasformò la Macedonia da periferia emarginata a centro nevralgico del mondo greco e divenne il protagonista assoluto e indiscusso di tutti gli eventi che in esso si svolsero» (p. 7). Nell'affrontare questo denso periodo storico Landucci mostra una notevole attenzione verso le principali testimonianze storiografiche antiche relative a tale epoca, delle quali riporta frequentemente alcuni brani, e decide parallelamente di riproporre anche le argute riflessioni contenute nelle opere moderne più accurate esprimendo sempre grande equilibrio nel valutare ogni ipotesi plausibile e nel riportare, laddove possibile, le conclusioni da lei considerate più accettabili. 
Nel complesso la figura di Filippo che emerge da questo breve resoconto appare quella di un abile governatore dotato di eccellenti doti diplomatiche, di indiscutibili qualità militari e determinato a seguire fino in fondo un'acuta strategia politica finalizzata a potenziare lo stato macedone a danno delle altre potenze che si affacciavano sull'Egeo. In particolare, l'innato carisma mostrato dal sovrano nel motivare e guidare i soldati macedoni, la capacità di adottare rapidamente intelligenti decisioni politiche, di concepire un'elaborata strategia di riforme, nonché l'abilità di condizionare le sorti di ogni disputa o trattativa a suo vantaggio permisero a Filippo di ribaltare le sorti del regno argeade, che al momento della sua ascesa al trono rischiava la dissoluzione, e di estendere la sfera d'influenza macedone dalle coste sud-occidentali del Ponto Eussino ai litorali epiroti dell'Adriatico e al Peloponneso, concentrando per la prima volta in una sola figura le sorti dell'intero mondo greco e le ambiziose aspirazioni di rivalsa nei confronti del secolare nemico achemenide.

Ripercorrendo i punti salienti della gloriosa scalata del basileus al conseguimento dell'egemonia sulla Grecia, l'autrice narra l'intera epopea di Filippo fino alla creazione di una testa di ponte in Asia e all'improvviso assassinio del re in conseguenza di intrighi di palazzo difficili da chiarire. In seguito a tutte le straordinarie imprese da lui compiute non stupisce dunque che all'apice del suo potere Filippo fosse considerato, soprattutto per il suo naturale talento nel fondare il suo immenso potere su una vastissima rete di rapporti personali di cui egli stesso era contemporaneamente promotore e fulcro, come il più grande fra i sovrani d'Europa - una grandezza che risulta, ancora oggi, innegabile - e che la sua immagine fosse venerata in trono fra quelle dei dodici déi. Si trattava infatti del primo (ma non ultimo) uomo della koiné greca che, in funzione del proprio potere politico, ebbe l'audacia di associarsi apertamente alle divinità dell'Olimpo senza timore di suscitare feroci reazioni e anche per questo, come afferma in conclusione Landucci sine ira ac studio, «il mondo, dopo il suo regno, non fu mai più lo stesso» (p. 135). 